\title{
Différenciation, fonction et contrôle de l'ostéoblaste
}

\section{Pierre Marie}

\section{ADRESSE}

P. Marie: Inserm U. 349 affiliée Cnrs, Hôpital Lariboisière, 2 , rue Ambroise-Paré,

Les grandes étapes de l'ostéogenèse sont caractérisées par l'engagement, la prolifération et la différenciation de cellules souches pluripotentes, qui se différencient en ostéoblastes fonctionnels chargés de la synthèse et de la minéralisation de la matrice osseuse. Le développement de modèles cellulaires et génétiques a permis de mieux comprendre les mécanismes impliqués dans le recrutement, la différenciation, la fonction et l'apoptose de l'ostéoblaste. La différenciation des ostéoblastes nécessite l'expression coordonnée du facteur de transcription majeur Cbfa1/ R unx2 et d'autres facteurs de transcription tels que msx-2, DIx5 et Twist, qui contrôlent l'expression des gènes cibles au cours de l'ossification endochondrale et membranaire. L'ostéoblaste est contrôlé de façon complexe par des facteurs systémiques et locaux dont certains mécanismes d'action ont été identifiés. De nombreux dysfonctionnements du recrutement, de la différenciation, ou de la fonction de l'ostéoblaste sont à l'origine de maladies osseuses généralisées ou localisées. Le développement des recherches dans ce domaine pourrait permettre l'identification de gènes cibles régulateurs de l'ostéoblastogenèse et, à terme, de développer de nouvelles stratégies pour stimuler l'ostéoformation.

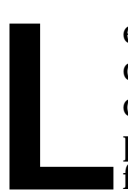

e tissu osseux est composé d'une matrice extracellulaire calcifiée dont les propriétés permettent d'assurer trois onctions principales: une fonction mécanique assurant le support du poids de l'organisme, une fonction de protection des organes essentiels, et une fonction métabolique liée à la capacité de stocker des minéraux, en particulier le calcium et le phosphate. Pour assurer ces fonctions, le tissu osseux est continuellement renouvelé par un processus de remodelage assuré par deux types cellulaires: les ostéoclastes qui résorbent la matrice osseuse et les ostéoblastes qui synthétisent une nouvelle matrice. Ce remodelage permet le maintien de la masse osseuse au cours de la vie adulte normale. Le déséquilibre de ce processus ou du couplage entre résorption et formation de l'os a des répercus- 
sions importantes sur l'architecture et la masse osseuse et peut conduire à long terme à une perte de l'intégrité de la structure du squelette et à des fractures, causes importantes de morbidité et de mortalité au cours du vieillissement. Le bon déroulement du remodelage nécessite des interactions étroites entre les cellules osseuses, la moelle osseuse et la matrice, interactions qui font appel à des facteurs solubles synthétisés par les cellules ou intégrés dans la matrice.

Le cycle de remodelage débute par une phase d'activation, caractérisée par la différenciation des ostéoclastes, suivie d'une phase de résorption de la matrice par les ostéoclastes mûrs (figure 1). A la suite de cette phase, les ostéoclastes se détachent, les précurseurs des ostéoblastes se différencient en pré-ostéoblastes puis en ostéoblastes mûrs qui synthétisent et déposent une nouvelle matrice qui comble la lacune de résorption. Le pré-ostéoblaste a une forme elliptique sans activité fonctionnelle, alors que l'ostéoblaste différencié est une cellule de forme cuboïde, avec un noyau apical, un cytoplasme basophile et une synthèse protéique intense. Une fois la matrice osseuse synthétisée et minéralisée, la majorité des cellules s'aplatissent et bordent la surface de l'os. Une partie des ostéoblastes subissent alors un phénomène d'apoptose, le reste se laissant inclure dans la matrice qu'ils ont synthétisées, devenant des ostéocytes reliés par un réseau d'extensions cytoplasmiques présentes dans des canalicules (figure 1).

Les processus cellulaires et moléculaires qui contrôlent la différenciation et la fonction de l'ostéoblaste sont encore mal connus. L'identification de ces processus intéresse autant les chercheurs fondamentalistes que les cliniciens qui sont à la recherche de stratégies thérapeutiques capables d'augmenter l'ostéoformation dans les maladies osseuses. Cet article résume nos connaissances de l'origine, de la différenciation, de la fonction et du contrôle de l'ostéoblaste.

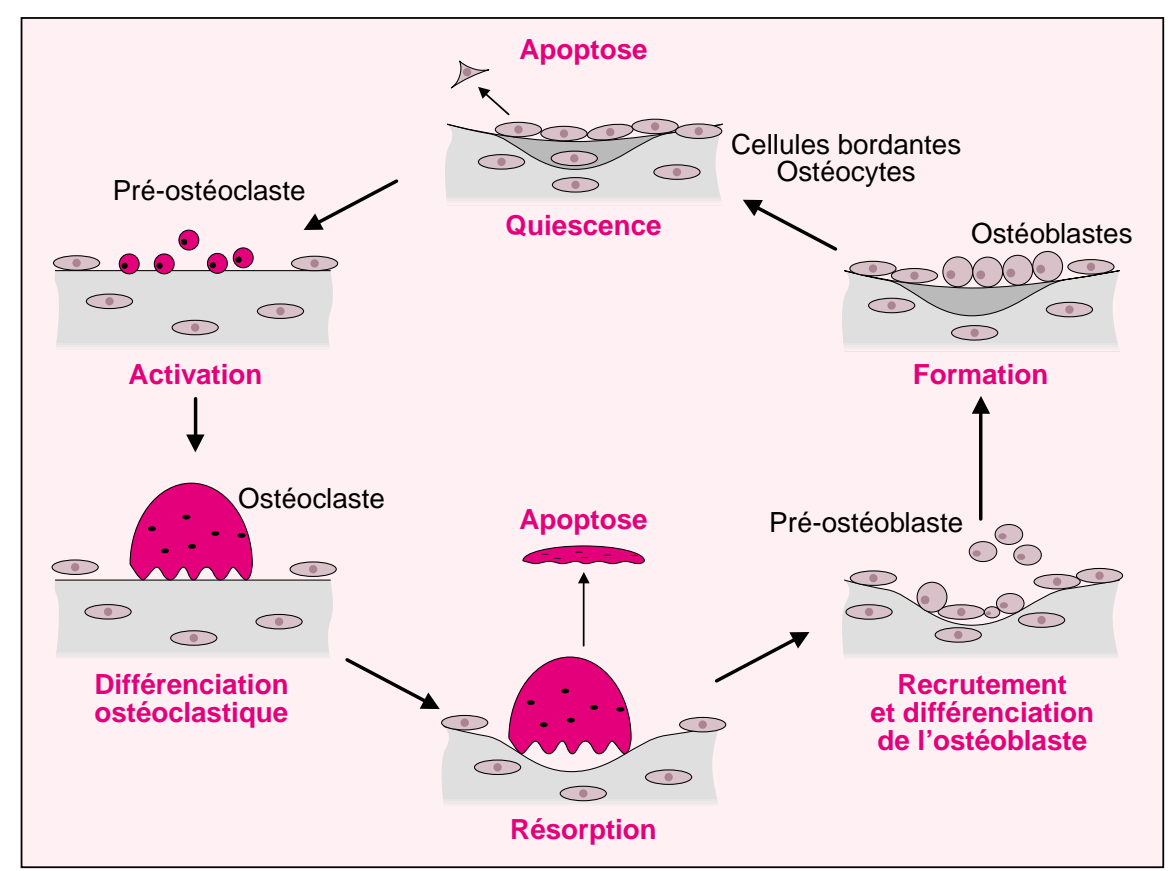

Figure 1. Place de l'ostéoblaste au cours du cycle de remodelage osseux. Le cycle de remodelage débute par une phase d'activation, caractérisée par la différenciation des ostéoclastes, suivie d'une phase de résorption de la matrice par les ostéoclastes. À la suite de cette phase, les ostéoclastes se détachent et meurent par apoptose, puis les précurseurs des ostéoblastes se différencient en pré-ostéoblastes puis en ostéoblastes qui synthétisent une nouvelle matrice comblant la lacune de résorption. À la fin de cette phase de formation, les ostéoblastes deviennent des cellules bordantes, subissent un phénomène d'apoptose ou se laissent inclure dans la matrice en devenant des ostéocytes.

\section{Origine et plasticité de l'ostéoblaste}

L'ossification endochondrale responsable de la formation des os longs est caractérisée par le dépôt de matrice osseuse sur une matrice cartilagineuse, alors que l'ossification membranaire au niveau des os plats procède d'une apposition de matrice extracellulaire sécrétée par les ostéoblastes (voir I'article de P. Ducy, p.1242 de ce numéro). Cependant, les processus cellulaires et moléculaires impliqués dans ces deux types d'ostéogenèse ne sont pas fondamentalement différents. Au niveau des os plats, les ostéoblastes proviennent de la différenciation de cellules précurseurs mésenchymateuses dérivées de la crête neurale. La formation se fait alors par condensation du mésenchyme. Au niveau de l'endoste, les ostéoblastes proviennent de la différenciation de cellules souches du stroma médullaire, capables de se différencier en cellules cartilagineuses, osseuses, musculaires ou adipocytaires sous l'induction de facteurs locaux et systémiques [1]. Le renouvellement des ostéoblastes se fait à partir de cellules souches mésenchymateuses médullaires.

Ces cellules mésenchymateuses ont une certaine plasticité et peuvent se différencier en chondroblastes, ostéoblastes ou adipocytes [2]. La différenciation vers l'une ou l'autre voie implique l'expression temporelle de facteurs de transcription, dont certains sont identifiés. L'expression de Sox-9 induit la voie chondroblastique, et celle de MyoD induit la voie myoblastique. L'expression du peroxisome PPAR y2 (proliferator activated receptor $\gamma 2$ ) induit la différenciation adipocytaire alors que celle de Cbfa1/Runx2 est nécessaire pour la différenciation ostéoblastique. L'expression de ces facteurs détermine la plasticité celulaire (figure 2).

\section{Différenciation de l'ostéoblaste}

Le développement de modèles de cultures de cellules ostéoblastiques murines et humaines a facilité l'étude de I'ostéogenèse. Les modèles les plus courants utilisent des cellules ostéoblastiques dérivées des os du crâne, de l'endoste $[3,4]$, ou du stroma médullaire [5], cultivées dans 


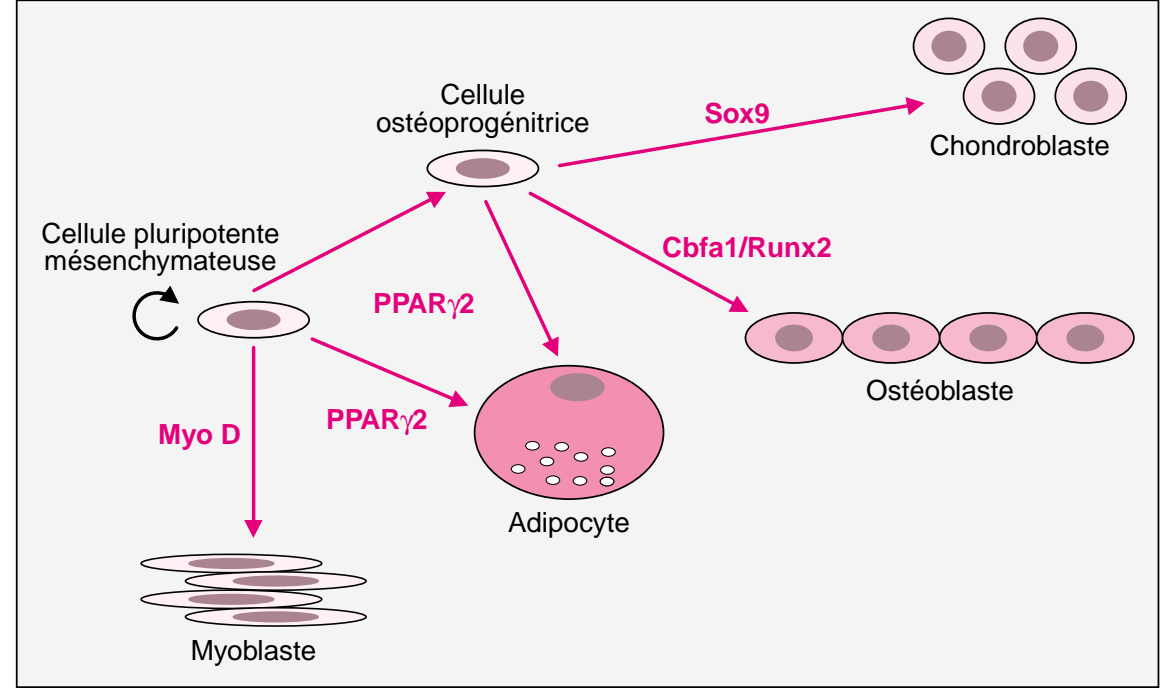

Figure 2. Les ostéoblastes proviennent de la différenciation de cellules souches mésenchymateuses pluripotentes. La différenciation vers un type de cellules donné nécessite l'expression de facteurs de transcription spécifiques. L'expression de MyoD induit la voie myoblastique. L'expression de Sox-9 induit la voie chondroblastique, celle de PPAR $\gamma 2$ induit la différenciation adipocytaire alors que l'expression de Cbfa/Runx2 est nécessaire pour la différenciation ostéoblastique.

des conditions permettant la synthèse d'une matrice osseuse qui se calcifie in vitro. Ces modèles ont permis d'identifier les grandes étapes du programme de différenciation de l'ostéoblaste et de déterminer la régulation de l'expression des gènes au cours de l'ostéogenèse in vitro [3-6].

L'ostéogenèse est caractérisée par l'engagement et la prolifération de cellules ostéoprogénitrices qui, après arrêt de la multiplication cellulaire, se différencient en ostéoblastes fonctionnels chargés de la synthèse et de la minéralisation de la matrice osseuse. Plusieurs marqueurs sont exprimés de façon séquentielle au cours de la différenciation ostéoblastique. Ce sont soit des marqueurs précoces de type facteur de transcription comme Cbfa1/ Runx2, soit des marqueurs fonctionnels. La phase de prolifération est associée à l'expresion de gènes précoces ( $\mathrm{c} f \mathrm{fos}$, histone $H 4$ ). Puis la phase de maturation est caractérisée par l'expression de gènes ostéoblastiques et associés à la production de matrice (phosphatase alcaline, collagène de type I, ostéopontine, tran sforming growth factor $\beta$ [TGF- $\beta$ ], fibronectine). Les ostéoblastes différenciés expriment des marqueurs tardifs de la différenciation (ostéocalcine, sialoprotéine osseuse) au début de la

\section{Fonctions de l'ostéoblaste}

La fonction principale de l'ostéoblaste est de synthétiser et de minéraliser la matrice osseuse au cours de la expression; --- : forte expression). croissance du squelette, du renouvellement de la matrice osseuse chez l'adulte et de la réparation osseuse tout au long de la vie. La matrice osseuse est composée majoritairement de collagène de type I dont le rôle est d'assurer la résistance et I'élasticité de l'os, propriétés dépendantes de la quantité et de la qualité du collagène synthétisé. Les ostéoblastes synthétisent également un grand nombre de protéines matricielles (fibronectine, ostéopontine, ostéonectine, ostéocalcine, sialoprotéine osseuse) dont le rôle n'est pas complètement connu. La présence de séquences de type RGD dans la fibronectine (abondante dans l'os immature), le collagène, I'ostéopontine et la sialoprotéine reconnues par les intégrines permettent I'adhérence des cellules osseuses à la matrice [7]. Ainsi, l'invalidation du gène de l'ostéopontine induit une augmentation de la masse osseuse en diminuant I'adhérence des ostéoclastes à la matrice et donc la résorption osseuse [8]. Les liaisons intégrines-protéines osseuses jouent aussi un rôle important dans la différenciation ostéoblastique [9]. Ainsi, la sur-expression d'un dominant négatif de l'intégrine $\beta 1$ induit un défaut de formation osseuse et, par la suite,

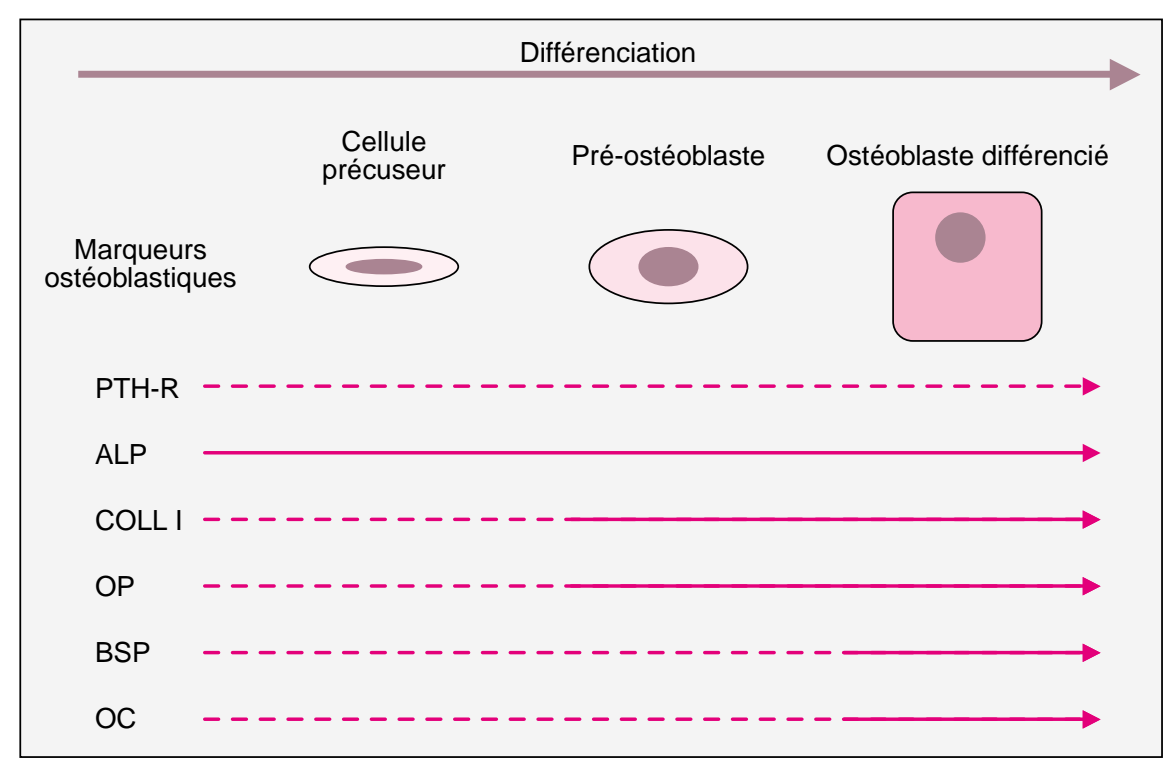

Figure 3. Principaux marqueurs exprimés au cours de la différenciation de l'ostéoblaste. La différenciation progressive de l'ostéoblaste est caractérisée par l'expression de gènes ostéoblastiques précoces (PTH-R: récepteur de la PTH; ALP : phosphatase alcaline; COLL-I : collagène de type I; OP : ostéopontine) ou tardifs (BSP: sialoprotéine osseuse; OC; ostéocalcine) (---: faible 
une ostéopénie chez la souris [10]. Les ostéoblastes synthétisent également des protéoglycanes, dont certains (biglycane, décorine) sont inclus dans la matrice [7]. Leur rôle serait de contrôler l'activité de facteurs locaux, tels que le TGF $\beta$. C'est ainsi que l'invalidation du gène codant pour le biglycane chez la souris a révélé l'induction d'une diminution de la formation osseuse et une ostéopénie [11]. Enfin, l'ostéoblaste contrôle aussi la dégradation de la matrice osseuse par la production de collagénases. Ainsi, la délétion d'une métalloprotéinase, la MT1-MMP induit un retard de croissance et une ostéopénie chez la souris [12]. Inversement, l'abolition du clivage du collagène de type I induit une augmentation de la formation osseuse [13], ce qui suggère que la dégradation de la matrice par l'ostéoblaste influence directement ou indirectement l'ostéogenèse.

La fonction finale de l'ostéoblaste mûr est de contribuer à la minéralisation de la matrice osseuse, ce qui nécessite un apport suffisant de minéraux (calcium et phosphate). Le transfert du calcium du milieu extracellulaire aux sites de nucléation présents dans le collagène se fait à la fois par diffusion passive et par transfert actif grâce à des pompes à calcium présentes dans la membrane de l'ostéoblaste. Les ions phosphate sont aussi transférés passivement et activement dans l'ostéoblaste à partir de la matrice par l'intermédiaire d'un système de transport de phosphate dépendant du sodium. Un autre mécanisme implique les phosphatases alcalines de l'ostéoblaste, qui dégradent les pyrophosphates inorganiques, inhibiteurs de la calcification, augmentant ainsi les taux de phosphate nécessaires à la minéralisation. L'invalidation de la phosphatase alcaline chez la souris induit d'ailleurs une anomalie de la minéralisation, ce qui souligne le rôle présumé de cette enzyme dans le processus de minéralisation [14]. De plus, certaines protéines osseuses telles que l'ostéopontine, l'ostéonectine, la sialoprotéine osseuse et des phosphoprotéines ont des sites de liaison au calcium, ce qui peut contribuer à la mise en route du processus de minéralisation de la matrice osseuse [7]. Enfin, l'ostéoblaste et ses précurseurs présents dans le stroma médullaire ont également pour rôle la production de molécules régulatrices solubles (OPG, RANKL) et de cytokines (M-CSF, TNF $\alpha, I L-1, I L-6, I L-11)$ relarguées dans le milieu extracellulaire et agissant au niveau des cellules précurseurs des ostéoclastes [15] (voir l'article de R. Baron, p. 1260 de ce numéro). Cette régulation joue un rôle majeur dans le contrôle de la résorption ostéoclastique et de la masse osseuse.

\section{Contrôle de l'ostéoblaste}

La formation osseuse est principalement dépendante du nombre d'ostéoblastes différenciés, et la différenciation ostéoblastique est sous le contrôle de facteurs de transcription, d'interactions cellulaires et matricielles et de facteurs systémiques et locaux.

\section{Rôle des facteurs de transcription}

Cbfal/ Runx2 est un facteur essentiel de l'engagement et de la différenciation des ostéoblastes. Ce facteur, membre de la famille de facteurs de transcription caractérisés par un domaine de liaison à l'ADN homologue de type runt/ Cbfa, se lie à un site consensus présent dans le promoteur de nombreux gènes dont la chaîne $\alpha 1$ du collagène de type I, la sialoprotéine osseuse, l'ostéocalcine, et l'ostéopontine. Cbfal est essentiel à l'ostéoformation in vivo et dans le maintien de la fonction ostéoblastique au cours de la croissance postnatale (voir l'article de P. Ducy, p. 1242 de ce numéro)

D'autres facteurs jouent un rôle important dans le contrôle de l'ostéogenèse. Ainsi, certains membres de la famille AP-1, dont c-fos, contrôlent la prolifération ostéoblastique et l'expression des gènes dont le promoteur présente des sites AP-1 [16]. La sur-expression de deltaFosB [17] ou de Fra-1 qui n'a pas de domaine de transactivation [18] induit une augmentation de la différenciation ostéoblastique, de la formation et de la masse osseuse chez la souris. Un autre facteur de transcription, Dlx-5, joue un rôle essentiel car l'invalidation du gène de DIX-5 chez la souris induit un retard d'ossification, sans altérer la morphogenèse [19]. Sa fonction pourrait être de contrôler l'expres- sion de la sialoprotéine osseuse et de l'ostéocalcine en interférant avec un autre facteur, Msx2. La sur-expression de Msx2 accélère I'ossification membranaire alors que son inactivation retarde la différenciation ostéoblastique, suggérant que Msx2 est un régulateur positif de l'ostéogenèse [20]. A l'inverse, le facteur de transcription Twist est un régulateur négatif de l'ostéoformation car son inactivation fonctionnelle augmente la prolifération et la différenciation des ostéoblastes [21] (voir l'article de V. El Ghouzzi et al., p. 1281 decenuméro). H iérarchiquement, l'engagement, la prolifération et la différenciation des ostéoblastes nécessite l'expression coordonnée du facteur de transcription majeur Cbfal/ Runx2 et d'autres facteurs régulateurs tels que msx-2, DIx5, Twist, qui contrôlent l'expression des gènes cibles au cours de l'ossification endochondrale et membranaire (figure 4).

\section{Rôle des interactions cellulaires}

Plusieurs types d'interactions cellulaires ont un rôle inducteur ou permissif sur la différenciation ostéoblastique. C'est le cas de certaines molécules d'adhérence intercellulaire telle que la $\mathrm{N}$-cadhérine qui est impliquée dans l'induction des gènes de différenciation, dont Cbfal/ Runx2, dans les ostéoblastes [22]. D'ailleurs, la sur-expression d'un dominant négatif de la $\mathrm{N}$-cadhérine supprime la différenciation ostéoblastique [23]. Les jonctions communicantes de type gap formées par les connexines contrôlent également l'activité fonctionnelle des ostéoblastes. Ainsi, la sur-expression des connexines 43 et 45 module l'expression de l'ostéocalcine et de la sialoprotéine osseuse dans les ostéoblastes [24]. In vivo, l'invalidation de la connexine 43 chez la souris altére la fonction ostéoblastique et induit un retard d'ossification [25], ce qui suggère que la communication intercellulaire assurée par ces protéines est nécessaire à la synthèse coordonnée des protéines osseuses par l'ostéoblaste in vivo.

\section{Rôle des facteurs systémiques}

Parmi les nombreuses hormones connues pour contrôler l'ostéofor- 


\begin{tabular}{|c|c|c|}
\hline $\begin{array}{c}\text { Séquence de différenciation } \\
\text { ostéoblastique }\end{array}$ & $\begin{array}{l}\text { Principaux facteurs } \\
\text { de contrôle }\end{array}$ & $\begin{array}{l}\text { Principaux facteurs } \\
\text { de transcription impliqués }\end{array}$ \\
\hline $\begin{array}{l}\text { Cellule souche } \\
\text { mésenchymateuse }\end{array}$ & & \\
\hline $\begin{array}{l}\text { Engagement } \\
\text { vers la voie } \\
\text { ostéoblastique }\end{array}$ & $\begin{array}{l}\text { BMP, } \\
\text { glucocorticoîdes }\end{array}$ & Cbfa1 \\
\hline $\begin{array}{l}\text { Prolifération } \\
\text { cellulaire }\end{array}$ & $\begin{array}{l}\text { FGF, TGF } \beta \text {, } \\
\text { PTH/PTHrP }\end{array}$ & $\begin{array}{l}\text { Msx-2, c-fos, } \\
\text { Twist }\end{array}$ \\
\hline $\begin{array}{l}\text { Différenciation } \\
\text { cellulaire }\end{array}$ & $\begin{array}{l}\text { BMP, TGF } \beta \text {, IGF, } \\
\text { glucocorticoîdes, } \\
\text { vitamine D }\end{array}$ & $\begin{array}{l}\text { Cbfa1, Msx-2, } \\
\text { Dlx-5, AP-1, } \\
\text { Twist }\end{array}$ \\
\hline Apoptose & $\begin{array}{l}\text { BMP, FGF, PTH, } \\
\text { glucocorticoîdes }\end{array}$ & Msx-2, NFкB \\
\hline
\end{tabular}

Figure 4. Principaux facteurs contrôlant l'ostéoblaste au cours de l'ostéogenèse. L'engagement des cellules souches, la prolifération, la différenciation et la durée de vie des ostéoblastes sont contrôlés par des facteurs de transcription agissant sur l'expression de gènes cibles. Plusieurs facteurs hormonaux et locaux contrôlent directement ou indirectement l'ostéogenèse en agissant à différentes étapes de la différenciation ostéoblastique.

mation, I'hormone parathyroïdienne (PTH), les hormones sexuelles, les glucocorticoïdes et la vitamine D sont les plus importantes. La PTH a pour cible les ostéoblastes qui expriment le récepteur de la PTH/ parathyroid hormone related peptide (PTHrP), et a des effets anaboliques sur la croissance et la formation osseuse [26]. In vivo, l'administration intermittente de PTH ou l'activation du récepteur stimule la formation de I'os trabéculaire [27]. In vitro, la PTH stimule la prolifération des précurseurs ostéoblastiques en induisant l'expression de c-fos, et prolonge la vie de l'ostéoblaste en inhibant I'apoptose. Certains effets de la PTH sur les ostéoblastes pourraient être secondaires à l'induction de facteurs de croissance locaux comme l'IGF (insulin-like growth factor) ou le TGF- $\beta$. Les œstrogènes exercent des effets directs et indirects importants sur les ostéoblastes [28]. Les ostéoblastes et leurs précurseurs expriment des récepteurs des œstrogènes de type $\alpha$ et $\beta$, le récepteur $\beta$ étant exprimé de façon majoritaire au cours de la différenciation ostéoblastique. Le rôle de ces récepteurs dans le contrôle de l'ostéogenèse reste cependant à établir. In vivo, l'invalidation du gène codant pour le récepteur $\alpha$ chez la malies osseuses alors que celle du gène codant pour le récepteur $\beta$ n'induit pas de perte osseuse trabéculaire mais plutôt une augmentation de la masse osseuse corticale. In vitro, les œstrogènes augmentent la prolifération et la différenciation des ostéoblastes bien que ces effets varient en fonction de l'état de différenciation et du type de récepteur exprimé [31]. Les effets sur la prolifération des précurseurs sont en partie dus à une augmentation de la production de facteurs locaux tels que le son (IGFBP). La régulation transcriptionelle des œstrogènes dans les ostéoblastes implique des facteurs de transcription tels que c-fos, NF-kB et $C / E B P-\beta$. De plus, les œstrogènes ont des effets anti-apoptotiques sur les ostéoblastes qui sont indépendants de l'activité transcriptionnelle de leurs récepteurs [29].

La 1,25(OH $)_{2}$-dihydroxyvitamine D3 - la forme active de la vitamine D - a également des effets physiologiques importants sur la différenciation ostéoblastique [30]. La 1,25( OH $)_{2-}$ dihydroxyvitamine D3 inhibe la transcription de la chaîne $\alpha 1(I)$ du collagène de type I et stimule l'expression de gènes ostéoblastiques tels que l'ostéopontine et l'ostéocalcine. Ce dernier effet implique Cbfal TGF- $\beta$, I'IGF et ses protéines de liai- qui contrôle le promoteur de l'ostéocalcine. In vivo, l'invalidation du gène codant pour le récepteur de la vitamine $D$ (VDR) chez la souris induit une diminution de la formation osseuse [31]. En revanche, la surexpression ciblée du VDR dans les ostéoblastes différenciés induit une augmentation de la masse osseuse corticale qui résulte d'une augmentation de la formation de l'os au niveau du périoste [32]. Ces données suggèrent un effet anabolique important de la vitamine D sur l'ostéogenèse in vivo.

Les glucocorticoïdes ont des effets multiples et complexes sur les ostéoblastes qui varient selon leur stade de maturation [33]. La dexaméthasone inhibe la prolifération des pré-ostéoblastes, induisant à long terme une diminution de la formation osseuse in vivo. En revanche, les glucocorticoïdes augmentent la différenciation des précurseurs ostéoblastiques dans le stroma médullaire. Outre cet effet pro-différenciateur, les glucocorticoïdes stimulent l'apoptose, ce qui diminue la durée de vie des ostéoblastes [34]. Par ailleurs, contrairement aux œstrogènes, les glucocorticoïdes stimulent l'expression de RANKL et réduisent celle de I'OPG par les cellules stromales/ ostéoblastes, ce qui induit une augmentation de la différenciation des ostéoclastes [15].

D'autres hormones pourraient contribuer à la régulation des gènes exprimés par l'ostéoblaste. Ainsi, les ostéoblastes expriment un récepteur nucléaire orphelin, l'estrogen related receptor $\alpha(E R R \alpha)$ qui stimule I'expression des gènes ostéoblastiques et pourrait ainsi contribuer à maintenir l'expression de gènes cibles en l'absence $d$ 'œstrogènes [35]. Un autre récepteur nucléaire orphelin, le retinoic acid-related orphan receptor $\alpha(\mathrm{ROR} \alpha)$, exprimé dans les cellules ostéoformatrices, augmente l'expression de la sialoprotéine osseuse et réprime celle de l'ostéocalcine. Une invalidation de ce gène chez la souris engendre une diminution de la masse osseuse, ce qui suggère une implication de ce récepteur nucléaire dans le contrôle de l'ostéoformation [36]. On sait enfin que l'ostéoformation est réglée au niveau central par la leptine (voir l'article de G. Karsenty, p. 1270 de ce numéro). 


\section{Rôle des facteurs locaux}

Plusieurs facteurs locaux sont d'importants régulateurs du recrutement, de la différenciation et de la fonction de l'ostéoblaste [1]. Ces facteurs peuvent agir en tant que facteurs autocrines ou paracrines ou servir de relais pour les hormones et les sollicitations mécaniques. Les facteurs les plus importants sont ceuxproduits par les ostéoblastes et qui s'incorporent dans la matrice osseuse, c'est-à-dire les IGF, le TGF- $\beta$, les BMP (bone morphogen etic proteins) et les FGF (fibroblast growth factors). L'IGF-I active la prolifération ostéoblastique et la synthèse de collagène de type I in vitro et la formation osseuse in vivo. De plus, I'IGF-I diminue l'apoptose des ostéoblastes. La sur-expression dirigée d'IGF-I dans les ostéoblastes induit une augmentation de l'activité, mais pas de la prolifération des ostéoblastes, ce qui conduit à une augmentation de la masse osseuse chez la souris [37]. Inversement, I'invalidation du gène IRS1 (insulin receptor substrate-1), impliqué dans la signalisation de I'IGF-I, induit une ostéopénie sévère qui résulte d'une réduction de la prolifération et de la différenciation des ostéoblastes [38], ce qui souligne l'importance de l'IGF-I dans le contrôle du recrutement et de la fonction de l'ostéoblaste. On sait que I'activité biologique des IGF est dépendante de leur liaison aux IGFBP (IGF-binding proteins), principalement I'IGFBP-3 et I'IGFBP-5, protéines produites par les ostéoblastes, incluses dans la matrice osseuse et contrôlées par des protéases spécifiques. D'ailleurs, il a été récemment établi que I'IGF-BP-5 a des effets directs sur l'ostéogenèse chez la souris [39]. Tout comme les IGF, les IGFBP sont contrôlées par la 1,25dihydroxyvitamine $D$, les œstrogènes et la PTH, ce qui souligne l'importance de ces facteurs de régulation dans le contrôle de la formation osseuse.

Le TGF- $\beta$ est un facteur local fondamental dans le contrôle de l'ostéogenèse. Le TGF- $\beta$ est produit sous forme latente par les ostéoblastes, puis stocké dans la matrice osseuse en association avec une protéine porteuse (LTBP), l'ensemble étant libéré lors de la résorption osseuse par les ostéoclastes. In vivo, le TGF- $\beta$ stimule fortement la formation osseuse chez le rat. La surexpression de TGF- $\beta 2$ chez la souris augmente la formation osseuse, probablement par ses effets stimulateurs directs de la fonction de l'ostéoblaste [40]. In vitro, le TGF- $\beta$ stimule la prolifération des pré-ostéoblastes ainsi que la production de collagène de type I et de I'ostéopontine. De plus, le TGF- $\beta$ inhibe la dégradation de la matrice en inhibant l'activité de la collagénase. En complément de ces effets, le TGF- $\beta$ a un effet anti-apoptotique sur les ostéoblastes [34]. En dehors de ces effets anaboliques, le TGF- $\beta$ contrôle la différenciation ostéoclastique en inhibant la production d'ODF et en augmentant celle de I'OPG par les cellules stromales et les ostéoblastes [15].

Les BMP (BMP-2, -4, -6, 7), membres de la famille du TGF- $\beta$, sont produites par les ostéoblastes et jouent un rôle fondamental dans le contrôle de la formation osseuse. Les BMP agissent à plusieurs étapes, en augmentant l'expression de Cbfal/ Runx2 dans les précurseurs ostéoblastiques et en stimulant l'expression des gènes ostéoblastiques (phosphatase alcaline, collagène de type I, ostéocal cine) dans les ostéoblastes [41]. Contrairement aux autres BMP, le BMP-3 inhibe la différenciation des cellules ostéoprogénitrices induite par la BM P-2, et l'invalidation du gène codant augmente la masse osseuse chez la souris [42]. Fait intéressant, il a été montré qu'une stimulation locale de l'expression de BMP-2 par des statines dans les ostéoblastes conduit à une augmentation de l'ostéoformation chez la souris [43], ce qui pourrait ouvrir de nouvelles voies thérapeutiques potentielles pour stimuler l'ostéoformation.

Les voies de signalisation impliquées dans les effets cellulaires des BMP sont maintenant mieux connues [41]. Les BMP se lient au récepteur de type II (BMPR-II) qui se dimérise avec le récepteur de type I (BMP-RI), ce qui induit la phosphorylation de protéines Smad, formant des complexes qui sont transloqués dans le noyau, reconnaissent une séquence sur le promoteur de gènes cibles. La différenciation des cellules mésenchymateuses précurseurs vers la lignée ostéoblastique, chondroblas- tique ou adipocytaire dépend d'ailleurs de l'expression des récepteurs de type I ou II. Ces voies de signalisation sont contrôlées positivement et négativement à différents niveaux. Ainsi, le TGF- $\beta$ et la BMP-2 contrôlent les taux d'expression des Smad et leur phosphorylation. De plus, des protéines de type hedgehog sont capables de coopérer avec les BMP pour régler la différenciation ostéoblastique aux stades précoces de l'ostéogen èse. D'autres protéines, comme noggin ou gremlin, induites par la BMP-2 réduisent sa propre activité dans les ostéoblastes. Enfin, une protéine appelée Tob, capable de s'associer à Smad1, 5 et 8, contrôle négativement l'activité de la BMP-2 in vitro et in vivo dans les ostéoblastes, ce qui montre la complexité de la régulation des effets des BMP dans l'ostéoblaste.

Les FGF sont des régulateurs importants de la prolifération, de la fonction et de l'apoptose des ostéoblastes [44]. In vivo, les FGF-1 et FGF-2 stimulent la formation osseuse de l'endoste et du périoste chez le rat. Inversement, l'invalidation du gène FGF-2 chez la souris diminue la formation osseuse et induit une ostéopénie [45]. In vitro, les effets des FGF dépendent du stade de différenciation cellulaire. Les FGF stimulent la prolifération ostéoblastique mais inhibent l'expression de plusieurs gènes ostéoblastiques dans les préostéoblastes. En revanche, un traitement à long terme par le FGF-2 augmente la population de cellules préostéoblastiques et l'ostéogenèse. Bien que le FGF-2 module l'expression des gènes ostéoblastiques, une partie de ces effets anaboliques sont indirects, induits par une stimulation de la production de TGF- $\beta$, d'IGF-I, d'IGF-II et d'IGFBP par les ostéoblastes. L'activité biologique des FGF depend de leur liaison à leur récepteur de haute affinité, les FGFR (FGF receptors), et des mutations activatrices des FGFR induisent une ostéogenèse prématurée qui implique une augmentation de la différenciation des ostéoblastes [46], ce qui démontre l'importance des interactions FGF/ FGFR dans le contrôle de l'ostéoblaste.

Ainsi, il apparaît clairement que l'ostéoformation est sous le contrôle de facteurs agisssant au niveau de 
I'engagement des cellules mésenchymateuses vers la voie ostéoblastique (BMP et ses protéines modulatrices), de la prolifération des pré-ostéoblastes (TGF- $\beta, F G F$ ), de la différenciation en ostéoblastes (BMP, IGF), de la régulation de la fonction (IGF, TGF- $\beta$ ), et enfin de la durée de vie des ostéoblastes (FGF, IGF, TGF- $\beta$, BMP) (figure 4).

\section{Dysfonctionnement ostéoblastique et maladies osseuses}

De nombreux dysfonctionnements du recrutement, de la différenciation, ou de la fonction de l'ostéoblaste conduisent au développement de maladies osseuses généralisées ou localisées [47]. Au cours du vieillissement, le nombre et l'activité des ostéoblastes diminuent, ce qui ne permet pas le remplacement de la matrice résorbée et induit à long terme une perte osseuse irréversible au niveau trabéculaire et cortical. L'immobilisation et I'hypokinésie sont également connues pour induire une diminution de la formation osseuse et une ostéopénie, en inhibant le recrutement et la différenciation de l'ostéoblaste. Par ailleurs, une variation de production endogène de PTH ou d'œstrogènes apparaissant lors de maladies métaboliques affectent le nombre, l'activité et la durée de vie des ostéoblastes. Enfin, de nombreuses mutations génétiques influencent de façon directe ou indirecte la fonction de l'ostéoblaste et l'ostéogenèse. Dans l'ostéogenèse imparfaite, des mutations affectant les chaînes $\alpha$ de collagène de type I diminuent la qualité de la matrice et conduisent à une fragilité osseuse sévère. Dans l'ostéopétrose, des mutations de gènes diminuant l'activité de l'ostéoclaste peuvent aussi affecter la différenciation de l'ostéoblaste [48]. D'autres mutations affectant des voies de signalisation dans l'ostéoblaste peuvent être à l'origine de maladies osseuses localisées. Par exemple, une activation constitutive de la protéine Gs $\alpha$ dans le syndrome de McCuneAlbright induit une augmentation de la prolifération des précurseurs ostéoblastiques, une diminution de leur différenciation, et une dysplasie fonctionnement du recrutement, de la différenciation ou du fonctionnement de l'ostéoblaste peut conduire à des anomalies de l'ostéogenèse et à une pathologie osseuse.

\section{Conclusions}

Le développement de modèles cellulaires et génétiques a permis de mieux comprendre les mécanismes impliqués dans le recrutement, la différenciation, la fonction et l'apoptose de l'ostéoblaste. Le rôle des facteurs de transcription, des interactions cellulaires et matricielles, et des facteurs systémiques et locaux dans le contrôle de l'ostéogenèse est maintenant mieux connu. Cependant, il reste beaucoup à apprendre sur les mécanismes cellulaires et moléculaires qui contrôlent la différenciation de l'ostéoblaste, en particulier chez I'homme. II est probable que le développement de nouveaux modèles d'étude cellulaire chez I'homme, de l'approche génomique et des études génétiques chez la souris permettront d'identifier de nouveaux mécanismes de régulation de I'ostéoblaste. Ces nouvelles voies de recherche pourraient permettre d'identifier de nouveaux gènes cibles régulateurs de l'ostéoblastogenèse et de développer à plus long terme des stratégies thérapeutiques capables de stimuler l'ostéoformation et d'augmenter la masse et la résistance osseuses dans les ostéopénies chez l'adulte

\section{RÉFÉRENCES}

1. De Vernejoul MC, Marie PJ. Cellules osseuses et remodelage osseux. In: Kuntz $D$ ed. M adadies métaboliques osseuses de l'adulté. Paris: Flammarion, 1996: 3-16.

2. Pittenger MF, Mackay AM, Beck SC, et al. Multilineage potential of adult human mesenshymal stem cells. Science 1999; 284 : 143-7.

3. Aubin JE, Liu F. The osteoblast lineage. In: Bilezikian JP, Raisz LG, Rodan GA, eds. Principles of bone biology. New York: Academic Press, 1996: 51-68.

4. Stein GS, Lian JB. Molecular mechanisms mediating proliferation/ differentiation interrelationships during progressive development of the Osteoblast phenotype. Endocrinol Rev 1993;14: 424-42.
5. Triffitt JT. The stem cell of the osteoblast. In : Bilezikian JP, Raisz LG, Rodan GA, eds. Principles of bone biology. New York: Academic Press, 1996: 39-50.

6. Marie PJ. O steoblasts and bone formation. In: Zaidi $M$, ed. Advances in organ biology: molecular and cellular biology of bone. Stamford, CT (USA) : JAI Press, 1999; 5B : 401-27.

7. Gehron Robey P. The biochemistry of bone. Endocrinol M etab Clin North Am 1989 ;18: 858-902.

8. Yoshitake H, Rittling SR, Denhardt DT, Noda M. Osteopontin-deficient mice are resistant to ovariectomy-induced bone resorption. Proc Natl Acad Sci USA 1999; 96 : 8156-60.

9. Franceschi RT. The developmental control of osteoblast-specific gene expression: role of specific transcription factors and the extracellular matrix environment. Crit Rev Oral Biol M ed 1999; 10: 40-57.

10. Zimmerman $D$, Jin $F$, Leboy $P, H$ ardy $S$, Damsky C. Impaired bone formation in transgenic mice resulting from altered integrin function in osteoblasts. Dev Biol 2000 ; 220: $2-15$

11. X u T, Bianco P, Fisher LW, \& al. Targeted disruption of the biglycan gene leads to an osteoporosis-like phenotype in mice. $\mathrm{N}$ at Genet 1998; 20: 78-82.

12. H olmbeck K, Bianco P, Caterina J, et al. MT 1-MMP-deficient mice develop dwarfism, osteopenia, arthritis, and connective tissue disease due to inadequate collagen turnover. Cell 1999; 99: 81-92.

13. Zhao W, Byrne MH, Wang Y, Krane SM O steocyte and osteoblast apoptosis and excessive bone deposition accompany failure of collagenase cleavage of collagen. I Clin Invest $2000 ; 106$ : 941-9.

14. Wennberg $C$, Hessle $L$, Lundberg $P$, et al. Functional characterization of osteoblasts and osteoclasts from alkaline phosphatase knockout mice. I Bone $M$ iner Res 2000; 15: 1879-88.

15. Suda T, Takahashi N, U dagawa N, Jimi E, Gillespie MT, Martin TJ. Modulation of osteoclast differentiation and function by the new members of the tumor necrosis factor receptor and ligand families. Endocrinol Rev 1999; 20: 345-57.

16. Grigoriadis AE, Wang ZQ, Wagner EF. Fos and bone cell development: lessons from a nuclear oncogene. Trends $G$ en et $1995 ; 11$ : 436-41.

17. Sabatakos G, Sims NA, Chen J, et al. O verexpression of DeltaFosB transcription factor(s) increases bone formation and inhibits adipogenesis. N at M ed 2000; 6: 985-90.

18. Jochum W, David JP, Elliott $C$, et al. Increased bone formation and osteosclerosis in mice overexpressing the transcription factor Fra-1. N at M ed 2000; 6: 980-4.

19. Acampora D Merla GR, Paleari L e al. Craniofacial, vestibular and bone defects in mice lacking the distal-less-related gene DIx5. Development 1999; 126: 3795-809. 


\section{RÉFÉRENCES}

20. Liu YH, Tang Z, Kundu RK, et al. M sx2 gene dosage influences the number of proliferative osteogenic cells in growth centers of the developing murine skull: a possible mechanism for MSX 2-mediated craniosynostosis in humans. Dev Biol 1999; 205: 260-74.

21. Yousfi $M$, Lasmoles F, Lomri A, Delannoy Ph, Marie PJ. I ncreased bone formation and decreased osteocalcin expression induced by reduced Twist dosage in the SaethreChotzen syndrome. J Clin Invest 2001; 107: 1153-61.

22. Haÿ E, Lemonnier J, Modrowski D, Lomri A, Lasmoles F, Marie PJ. Bone morphogenetic protein-2 promotes human calvaria cell aggregation and osteoblast differentiation by increasing $\mathrm{N}$-and $\mathrm{E}$-cadherin expression. J Cell Physiol 2000; 183: 117-28.

23. Cheng SL, Shin CS, Towler DA, Civitelli R. A dominant negative cadherin inhibits osteoblast differentiation. J Bone $M$ iner Res 2000; 15: 2362-70.

24. Lecanda F, Towler DA, Ziambaras K, et al. Gap junctional communication modulates gene expression in osteoblastic cells. M ol Biol Cell 1998; 9: 2249-58.

25. Lecanda F, Warlow PM, Sheikh S, Furlan $F$, Steinberg TH, Civitelli R. Connexin43 deficiency causes delayed ossification, craniofacial abnormalities, and osteoblast dysfunction. J Cell Biol 2000; 151: 931-44.

26. Strewler GJ. Local and systemic control of the osteoblast. j Clin Invest 2001; 107: 271-2.

27. Calvi LM, Sims NA, Hunzelman JL, et al. Activated parathyroid hormone/ parathyroid hormone-related protein receptor in osteoblastic cells differentially affects cortical and trabecular bone. J Clin Invest 2001; 107: 277-86.

28. De Vernejoul MC, Marie PJ. Estrogènes et tissu osseux. M ed Ther 2001; 2,1: 29-33.

29. Kousteni S, Bellido T, Plotkin $\mathrm{LI}$, et al. Nongenotropic, sex-nonspecific signaling through the estrogen or androgen receptors: dissociation from transcriptional activity. Cell 2001; 104: 719-30.

30. Christakos $S$. Vitamin $D$ gene regulation. In : Bilezikian JP, Raisz LG, Rodan GA, eds. Principles of bone biology. New York: Academic Press, 1996: 435-46.

31. Yoshizawa $T, H$ anda $Y, U$ ematsu $Y$, et al. $M$ ice lacking the vitamin $D$ receptor exhibit impaired bone formation, uterine hypoplasia and growth retardation after weaning. $N$ at Gen et $1997 ; 16: 391-6$.

32. Gardiner EM, Baldock PA, Thomas GP et al. Increased formation and decreased resorption of bone in mice with elevated vitamin $D$ receptor in mature cells of osteoblastic lineage. FA SEB J 2000; 14: 1908-16.

33. Lukert BP, Kream BE. Clinical and basic aspects of glucocorticoid action in bone. In: Bilezikian JP, Raisz LG, Rodan GA, eds. $\mathrm{m} / \mathrm{s} \mathrm{n}^{\circ} 12$, vol. 17, décembre 2001
Principles of bone biology. New York: Academic Press, 1996: 533-48.

34. Manolagas SC. Birth and death of bone cells: basic regulatory mechanisms and implications for the pathogenesis and treatment of osteoporosis. Endocrinol Rev 2000; 21: 115-37.

35. Bonnelye $E$, Merdad L, Kung V, Aubin JE. The orphan nuclear estrogen receptorrelated receptor alpha (ERRalpha) is expressed throughout osteoblast differentiation and regulates bone formation in vitro. J Cell Biol 2001; 153 : 971-84.

36. Meyer T, Kneissel M, Mariani J, Fournier $B$. In vitro and in vivo evidence for orphan nuclear receptor RORalpha function in bone metabolism. Proc Natl Acad Sci USA 2000; 97: 9197-202.

37. Zhao G, Monier-Faugere MC, Langub $M C$ et al. Targeted overexpression of insulin-like growth factor I to osteoblasts to transgenic mice: increased trabecular bone volume without increased osteoblast proliferation. Endocrinology 2000; 141: 2674-82.

38. Ogata N, Chikazu D, Kubota N, \& al. Insulin receptor substrate-1 in osteoblast is indispensable for maintaining bone turnover. J Clin Invest 2000 ; 105 : 935-43.

39. Miyakoshi N, Richman C, Kasukawa $Y$, Linkhart TA, Baylink DJ, Mohan S. Evidence that IGF-binding protein-5 functions as a growth factor. J Clin Invest $2001 ; 107$ : 73-81.

40. Erlebacher A, Derynck R. Increased expression of TG'F-beta 2 in osteoblasts results in an osteoporosis-like phenotype. I Cell Biol 1996; 132: 195-210.

41. Yamaguchi A, Komori T, Suda T. Regulation of osteoblast differentiation mediated by bone morphogenetic proteins, hedgehogs, and Cbfal. Endocrinol Rev 2000; 21 : 393-411.

42. Daluiski A, Engstrand T, Bahamonde $\mathrm{ME}$, t al. Bone morphogenetic protein-3 is a negative regulator of bone density. Nat Genet 2001; 27: 84-8.

43. Mundy G, Garrett R, Harris S, et al. Stimulation of bone formation in vitro and in rodents by statins. Science 1999; 286: 1946-9.

44. Marie PJ, Debiais F, Lomri A, Lemonnier J. Fibroblast growth factors and osteoblasts. In: Canalis E, eds. Sketetal growth factors. New York: Lippincott, Williams and Wilkins 2000; 179-96.

45. Montero A, Okada Y, Tomita M, et al. Dis ruption of the fibroblast growth factor-2 gene results in decreased bone mass and bone formation. J Clin Invest 2000; 105: 1085-93.

46. Lomri $A$, Lemonnier $J$, Hott $M$, et al. Increased calvaria cell differentiation and bone matrix formation induced by fibroblast growth factor receptor-2 mutations in Apert syndrome. J Clin Invest 1998; 101: 1310-7.

47. Marie PI, de Vernejoul MC. Facteurs systémiques et locaux du remodelage osseux. In : Kuntz D, ed. M aladies métaboliques osseuses de l'adulte. Paris: Flammarion, 1996: 49-70.
48. Marzia M, Sims NA, Voit S, et al. Decreased c-Src expression enhances osteoblast differentiation and bone formation. J Cell Biol 2000; 151: 311-20.

49. Marie PJ, de Pollak C, Chanson P, Lomri A. Increased osteoblastic cell proliferation associated with activating $G_{s} \alpha$ mutation in monostotic and polyostotic fibrous dysplasia. Am J Pathol 1997; 150, 3: 1059-69.

\section{Summary}

Differenciation, function and regulation of osteoblast

Bone formation is a complex process involving the commitment and differentiation of osteoblasts, formation and mineralization of bone matrix. The recent development of gene manipulation in mice and studies of genetic mutations affecting the osteoblast in humans led to improve considerably our understanding of the processes controling the birth, life and death of osteoblasts. Several transcription factors are now known to control osteoblast recruitment and differentiation. Moreover, cell-cell and cell-matrix interactions mediated by transmembrane proteins have been shown to play an important role in osteogenesis. There is also now genetic evidence that bone matrix proteins themselves play a role in bone formation in vivo. Genetic studies also clarified the role of regulatory local and systemic factors in the control of bone formation, and allowed to identify several signaling pathways involved in osteoblast function. Finally, apoptosis was shown to be an important mechanism by which osteoblast lifespan and bone formation are controlled. It is expected that the rapid development of genetic studies will facilitate the identification of novel genes regulating osteoblasts, which may allow in the long term to develop strategies capable of increasing bone formation in the postnatal life.

\section{TIRÉS À PART}

P. Marie. 